\title{
Can a deep learning model based on intraoperative time-series monitoring data predict post-hysterectomy quality of recovery?
}

Xu Zhao ${ }^{1,2}$, Ke Liao ${ }^{3,4}$, Wei Wang ${ }^{3}$, Junmei $X u^{2^{*}}$ and Lingzhong Meng ${ }^{1 *}$ (1)

\begin{abstract}
Background: Intraoperative physiological monitoring generates a large quantity of time-series data that might be associated with postoperative outcomes. Using a deep learning model based on intraoperative time-series monitoring data to predict postoperative quality of recovery has not been previously reported.

Methods: Perioperative data from female patients having laparoscopic hysterectomy were prospectively collected. Deep learning, logistic regression, support vector machine, and random forest models were trained using different datasets and evaluated by 5 -fold cross-validation. The quality of recovery on postoperative day 1 was assessed using the Quality of Recovery-15 scale. The quality of recovery was dichotomized into satisfactory if the score $\geq 122$ and unsatisfactory if $<122$. Models' discrimination was estimated using the area under the receiver operating characteristics curve (AUROC). Models' calibration was visualized using the calibration plot and appraised by the Brier score. The SHapley Additive exPlanation (SHAP) approach was used to characterize different input features' contributions.

Results: Data from 699 patients were used for modeling. When using preoperative data only, all four models exhibited poor performance (AUROC ranging from 0.65 to 0.68). The inclusion of the intraoperative intervention and/or monitoring data improved the performance of the deep leaning, logistic regression, and random forest models but not the support vector machine model. The AUROC of the deep learning model based on the intraoperative monitoring data only was $0.77(95 \% \mathrm{Cl}, 0.72-0.81)$, which was indistinct from that based on the intraoperative intervention data only (AUROC, $0.79 ; 95 \% \mathrm{Cl}, 0.75-0.82$ ) and from that based on the preoperative, intraoperative intervention, and monitoring data combined (AUROC, $0.81 ; 95 \% \mathrm{Cl}, 0.78-0.83$ ). In contrast, when using the intraoperative monitoring data only, the logistic regression model had an AUROC of $0.72(95 \% \mathrm{Cl}, 0.68-$ 0.77), and the random forest model had an AUROC of 0.74 ( $95 \% \mathrm{Cl}, 0.73-0.76)$. The Brier score of the deep learning model based on the intraoperative monitoring data was 0.177 , which was lower than that of other models.

\footnotetext{
* Correspondence: xujunmei@139.com; lingzhong.meng@yale.edu

2Department of Anesthesiology, The Second Xiangya Hospital, Central South University, Changsha, Hunan Province, China

'Department of Anesthesiology, Yale University School of Medicine, 333

Cedar St, New Haven, CT 06520, USA

Full list of author information is available at the end of the article
}

(c) The Author(s). 2021 Open Access This article is licensed under a Creative Commons Attribution 4.0 International License, which permits use, sharing, adaptation, distribution and reproduction in any medium or format, as long as you give appropriate credit to the original author(s) and the source, provide a link to the Creative Commons licence, and indicate if changes were made. The images or other third party material in this article are included in the article's Creative Commons licence, unless indicated otherwise in a credit line to the material. If material is not included in the article's Creative Commons licence and your intended use is not permitted by statutory regulation or exceeds the permitted use, you will need to obtain permission directly from the copyright holder. To view a copy of this licence, visit http://creativecommons.org/licenses/by/4.0/ The Creative Commons Public Domain Dedication waiver (http://creativecommons.org/publicdomain/zero/1.0/) applies to the data made available in this article, unless otherwise stated in a credit line to the data. 
(Continued from previous page)

Conclusions: Deep learning based on intraoperative time-series monitoring data can predict post-hysterectomy quality of recovery. The use of intraoperative monitoring data for outcome prediction warrants further investigation.

Trial registration: This trial (Identifier: NCT03641625) was registered at ClinicalTrials.gov by the principal investigator, Lingzhong Meng, on August 22, 2018.

Keywords: Deep learning, Machine learning, Time-series monitoring data, Hysterectomy, Quality of recovery, Prediction

\section{Background}

Perioperative care has two fundamental goals. One is to reduce the incidence of complications, and the other is to enhance recovery to the greatest extent possible. Complications and quality of recovery are related but distinct phenomena (Jammer et al., 2015). Complications negatively impact recovery, while the quality of recovery can still vary among patients who, clinically, do not have any or have comparable complications (Bowyer, Jakobsson, Ljungqvist, \& Royse, 2014). The question is how to accomplish these goals. One solution is prognostication, i.e., if we are informed of the level of the risk for a given complication or the potential for an unsatisfactory recovery, we can adjust patient care based on the best evidence to minimize undesirable outcomes (Coulter, Locock, Ziebland, \& Calabrese, 2014). Therefore, these at-risk patients should receive enhanced care.

To guide intraoperative care, prognostication must happen before surgery or during surgery. Any prognostication based only on preoperative information misses intraoperative information, which could adversely affect prognostication as the quality of intraoperative care is one of the major determinants of postoperative outcomes (Ljungqvist, Scott, \& Fearon, 2017). It is theoretically ideal to incorporate intraoperative information during the prognostication of postoperative courses. To do so, practitioners must collect intraoperative information in real time, feed the data into validated models instantaneously, and use the output to guide intraoperative care in a timely manner (Mathis, Kheterpal, \& Najarian, 2018).

Intraoperative data can be categorized into two types: one is time-series monitoring data, such as heart rate and blood pressure, and the other is intervention data, such as medications and fluids given to patients. The time-series monitoring data carry temporal and dynamic information, a unique feature distinguishing themselves from non-time-series intervention data. However, there may be an association between intervention and timeseries monitoring data because intraoperative interventions may make a footprint in monitoring, for example, the administration of phenylephrine (i.e., an intervention) increases blood pressure and decreases heart rate (i.e., corresponding change in monitoring). We speculate that this footprint may sometimes make the simultaneous use of intervention and monitoring data in a prediction model redundant. Currently, determining how best to use the intraoperative time-series monitoring data during prognostication remains largely unknown.

During conventional modeling (e.g., logistic regression), processed parameters of the time-series monitoring data, such as the maximum, minimum, mean, and median values, are used in modeling. The concern regarding this approach is the loss of temporal and dynamic information embedded in the time-series data. Deep learning models can uniquely learn from the original time-series data, which may be superior to models that can only learn from processed parameters (Fawaz, Forestier, Weber, Idoumghar, \& Muller, 2019a).

In this study, we hypothesized that the InceptionTime deep learning model based on the intraoperative timeseries monitoring data can predict the quality of recovery after surgery. We based this study on data collected from the intervention guided by Muscular Oxygenation to Decrease the Incidence of PostOperative Nausea and Vomiting (iMODIPONV) trial. As a result, the data were derived from female patients having laparoscopic hysterectomy.

\section{Methods}

This study was based on data collected in the iMODIPONV trial conducted in female patients having laparoscopic hysterectomy (ClinicalTrials.gov Registration: NCT03641625) (Li et al., 2020). This study was conducted according to the Guidelines for Developing and Reporting Machine Learning Predictive Models in Biomedical Research (Luo et al., 2016).

\section{Patients}

Participants were 18-65-year-old females who had no history of smoking and were scheduled for elective laparoscopic hysterectomy. Their American Society of Anesthesiologists (ASA) physical status classifications were I-II. Patients who were scheduled for vaginal or open hysterectomy, urgent or emergent surgery, or a procedure involving bowel resection were excluded. Patients with major systemic comorbidities or who 
had undergone chemotherapy or radiotherapy within 3 months before surgery were also excluded.

\section{Data}

The modeling used preoperative, intraoperative intervention, and intraoperative monitoring data (Table 1). Preoperative data included patient demographics, ASA classification, anesthesia-relevant history, comorbidities, and laboratory results. Intraoperative intervention data included anesthetic time, medications, inputs, and outputs. The total of these variables for the entire surgery was used in modeling. Intraoperative monitoring data included time-series heart rate, blood pressure, respiratory rate, pulse oxygen saturation, end-tidal carbon dioxide, and body temperature. We additionally included muscular tissue oxygen saturation data as it was monitored in the iMODIPONV trial. All intraoperative monitoring data were recorded every 2 seconds by a research laptop. The recording started approximately $5 \mathrm{~min}$ before anesthesia induction and stopped at the end of surgery.

For time-series data, we regarded values that fell outside the 0.5th and 99.5th percentiles as outliers and treated them as missing data. The missing time-series data were filled using values corresponding to the immediately preceding time points. Time-series data varied with respect to recording duration due to variations in surgical time across patients. We scaled all time-series data to the same extent of 1000 time points using standard down-sampling or up-sampling methods (spline interpolation). We chose 1000 time points because our preliminary analyses indicated that models using 1000 time points had non-inferior performance and could be trained faster than otherwise (eTable 1 in Additional file 1).

In the deep learning model, we converted non-timeseries data in the form of a single value per feature per patient to time-series data by replicating the value across all time points. In all models, categorical data were converted into binary data using the one-hot encoding method (Potdar, Pardawala, \& Pai, 2017). Missing numerical data were filled using mean imputations. All continuous data, including time-series monitoring data, were normalized to a range from 0 to 1 . The upper and lower limits used in normalization are presented in eTable 2 in Additional file 1.

\section{Outcome definition}

In this study, we targeted the quality of recovery as an outcome measure, which was assessed using the Quality of Recovery-15 (QoR-15) scale on postoperative day 1 (Stark, Myles, \& Burke, 2013). The QoR-15 scale, ranging from 0 to 150 , is a validated patient-reported measure of the quality of recovery (Myles et al., 2018). We dichotomized the quality of recovery into satisfactory if the QoR-15 score $\geq 122$ and unsatisfactory if $<122$. This cutoff value was consistent with a previous study that

Table 1 Type and nature of the data used in modeling

\begin{tabular}{|c|c|}
\hline Type of data & Nature of data \\
\hline \multicolumn{2}{|l|}{ Preoperative data } \\
\hline Demographics (Age, height, body weight, and BMI) & Numerical data \\
\hline ASA physical status classification & Categorical data \\
\hline $\begin{array}{l}\text { Anesthesia-relevant history (general anesthesia, spinal anesthesia, nerve block or local anesthesia, } \\
\text { postoperative nausea and vomiting, and motor sickness) }\end{array}$ & Categorical data \\
\hline $\begin{array}{l}\text { Comorbidities (psychiatric disease, neurologic disease, hypertension, cardiovascular disease, pulmonary } \\
\text { disease, endocrinologic disease, renal insufficiency, and digestive disease) }\end{array}$ & Categorical data \\
\hline Laboratory results (hemoglobin, hematocrit, and creatinine) & Numerical data \\
\hline \multicolumn{2}{|l|}{ Intraoperative intervention data } \\
\hline Anesthetic time & Numerical data \\
\hline Propofol, remifentanil, and sufentanil ${ }^{b}$ & Numerical data \\
\hline Crystalloid, urine output, and blood loss & Numerical data \\
\hline \multicolumn{2}{|l|}{ Intraoperative monitoring data } \\
\hline Respiratory rate and end-tidal carbon dioxide & $\begin{array}{l}\text { Time-series data, captured from } \\
\text { anesthesia machine }\end{array}$ \\
\hline $\begin{array}{l}\text { Heart rate, systolic blood pressure, diastolic blood pressure, mean arterial pressure, pulse oxygen } \\
\text { saturation and body temperature }\end{array}$ & $\begin{array}{l}\text { Time-series data, captured from vital sign } \\
\text { monitors }\end{array}$ \\
\hline Muscular tissue oxygen saturation & $\begin{array}{l}\text { Time-series data, captured from NIRS- } \\
\text { based tissue oximeter }\end{array}$ \\
\hline
\end{tabular}

$B M I$ body mass index, ASA American Society of Anesthesiologists, NIRS near-infrared spectroscopy, iMODIPONV trial the intervention guided by Muscular Oxygenation to Decrease the Incidence of PostOperative Nausea and Vomiting (iMODIPONV) trial

${ }^{a}$ For the intraoperative intervention data, the totals of different variables for the entire surgery were used in modeling

${ }^{b}$ Sufentanil was the standardized opioid for pain control in the iMODIPONV trial 
categorized the quality of recovery as excellent or good if the QoR-15 score $\geq 122$, and moderate or poor if the QoR-15 score <122 (Kleif \& Gögenur, 2018). We also referenced the mean and median QoR-15 values of our patient population during the determination of the cutoff value.

\section{Model development}

Stratified, 5-fold cross-validation was used to develop training and testing sets (Fig. 1).

\section{Deep learning model}

The architecture of the deep learning model is presented in Fig. 2. The model was based on InceptionTime (Fawaz et al., 2019a, b), which ensembled six sequentially stacked deep convolutional neural network modules (Inception module). In each inception module, the multidimension time-series data were transformed into onedimension data (bottleneck). This process reduced the dimensionality of the time-series data and potentially avoided overfitting small datasets. Three one-dimension filters with lengths of 10,20, and 40 were applied simultaneously to the output of bottleneck (convolution). A parallel operation was performed to avoid the influence of small perturbations. A window with a length of 3 was slid onto the original multi-dimension time-series data, and the maximum value in this window was computed (MaxPooling). The outputs of each independent parallel convolution and MaxPooling were concatenated to form the output of the current Inception module. The Inception network classifier contained two different residual blocks to mitigate the vanishing gradient. Each residual block was comprised of three Inception modules. Ten percent of patients in the training set were reserved and used as a validation set during the training of the deep learning model. The binary cross-entropy loss with sigmoid layer was used as a loss function. To avoid model overfitting, the training process was stopped when the validation loss began to increase.

\section{Other models}

We compared the deep learning model to three widely used machine learning algorithms, including logistic regression, support vector machine, and random forest. Because these algorithms cannot handle the original time-series data, the maximum, minimum, mean, and standard deviation (SD) values of each time-series data were used in modeling. Default parameters of the scikit-

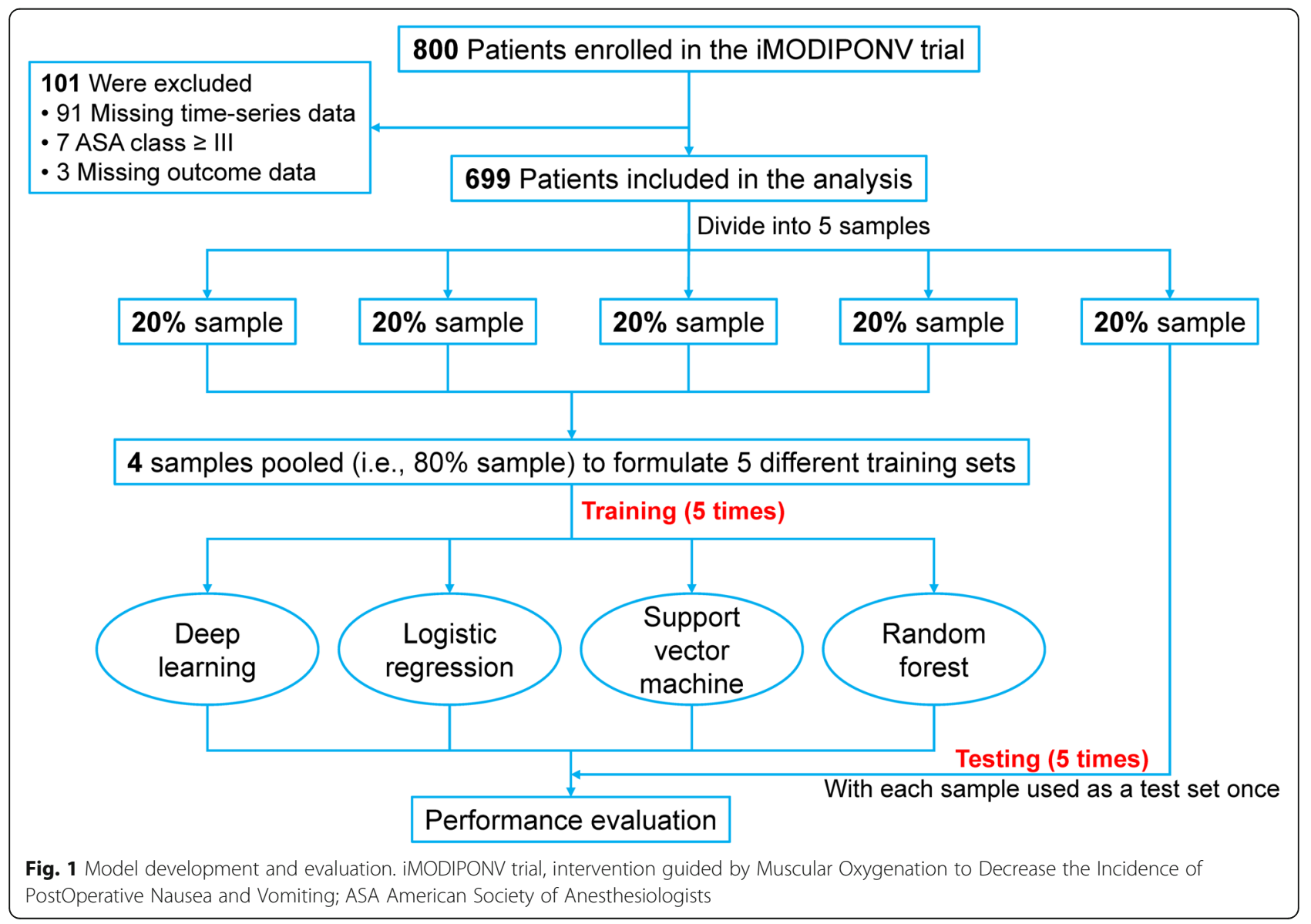




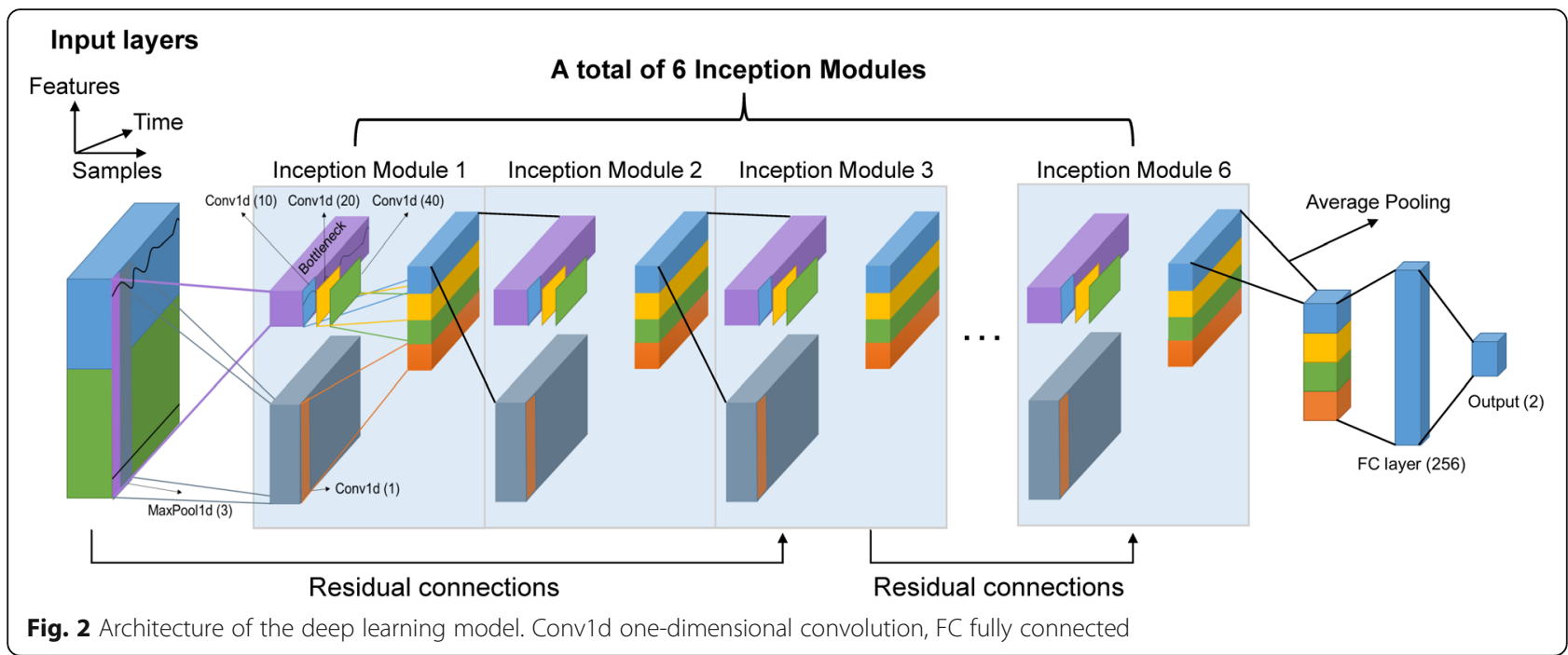

learn (version 0.22.2.post1) were used in the training process of these algorithms.

\section{Model performance}

Accuracy, sensitivity, specificity, F1 score, and area under the receiver operating characteristics curve (AUROC) were used to estimate model discrimination (Alba et al., 2017). Calibration (goodness of fit) was visualized using the calibration plot (Alba et al., 2017). Calibration reflects the extent to which the expected (predicted from the model) and observed outcomes agree. The calibration plot was graphically depicted using the observed outcome frequencies on the ordinate plotted against the expected outcome probabilities on the abscissa. The better the model was calibrated, the closer the points approximated the perfectly calibrated diagonal traveling from the bottom left to the top right in the graph. The overall agreement between the predicted and observed outcomes was quantified using the Brier score (Rufibach, 2010). The Brier score ranges from 0 to 1 and is the mean squared difference between the predicted and observed outcomes. A lower Brier score indicates improved model accuracy.

\section{Feature importance}

For the deep learning model, class activation mapping was used to visualize the contributions of different parts of time-series data to the prediction (Zhou, Khosla, Lapedriza, Oliva, \& Torralba, 2016). Class activation mapping provides visual explanation for convolutional neural networks by highlighting the significance of contribution based on local backpropagation. In this study, we used class activation mapping to explore whether any parts of the input appeared peculiar that might confuse the network.
For the logistic regression, support vector machine, and random forest models, the SHapley Additive exPlanation (SHAP) approach was used to appraise the significance of the contribution made by different input features to the prediction (Lundberg \& Lee, 2017). The SHAP method is based on the game theory approach that assigns each feature a SHAP value. A larger absolute SHAP value represents a bigger contribution made by the feature to the prediction. We used the fold that had the best prediction performance to evaluate feature importance.

\section{Software}

Model development and evaluation were performed using Python 3.6.9. The deep neural network models were developed using the PyTorch (version 1.0.1), timeseries (version 0.0.6), and fastai2 (version 0.0.19) modules. The logistic regression, support vector machine, and random forest models were developed using the scikit-learn module (version 0.22.2.post1). Performance metrics were calculated using the scikit-learn module (version 0.22.2.post1).

\section{Results}

\section{Patient characteristics}

The iMODIPONV trial enrolled 800 patients. Of these 800 patients, we excluded 101 patients including 94 patients due to missing monitoring or outcome data and 7 patients due to an ASA classification $\geq$ III (Fig. 1). A total of 699 patients (age, $50 \pm 7$ years; body mass index, $25 \pm 3$ $\mathrm{kg} / \mathrm{m}^{2}$ ) were included in this study (Table 2). The mean and median QoR-15 scores of our patients were 121 (19, SD) and 122 (109-135, IQR), respectively, and 50.6\% $(354 / 699)$ of our patients had a QoR-15 score $\geq 122$. The distribution of the QoR-15 score is presented in Fig. 3. 
Table 2 Perioperative data $(n=699)$

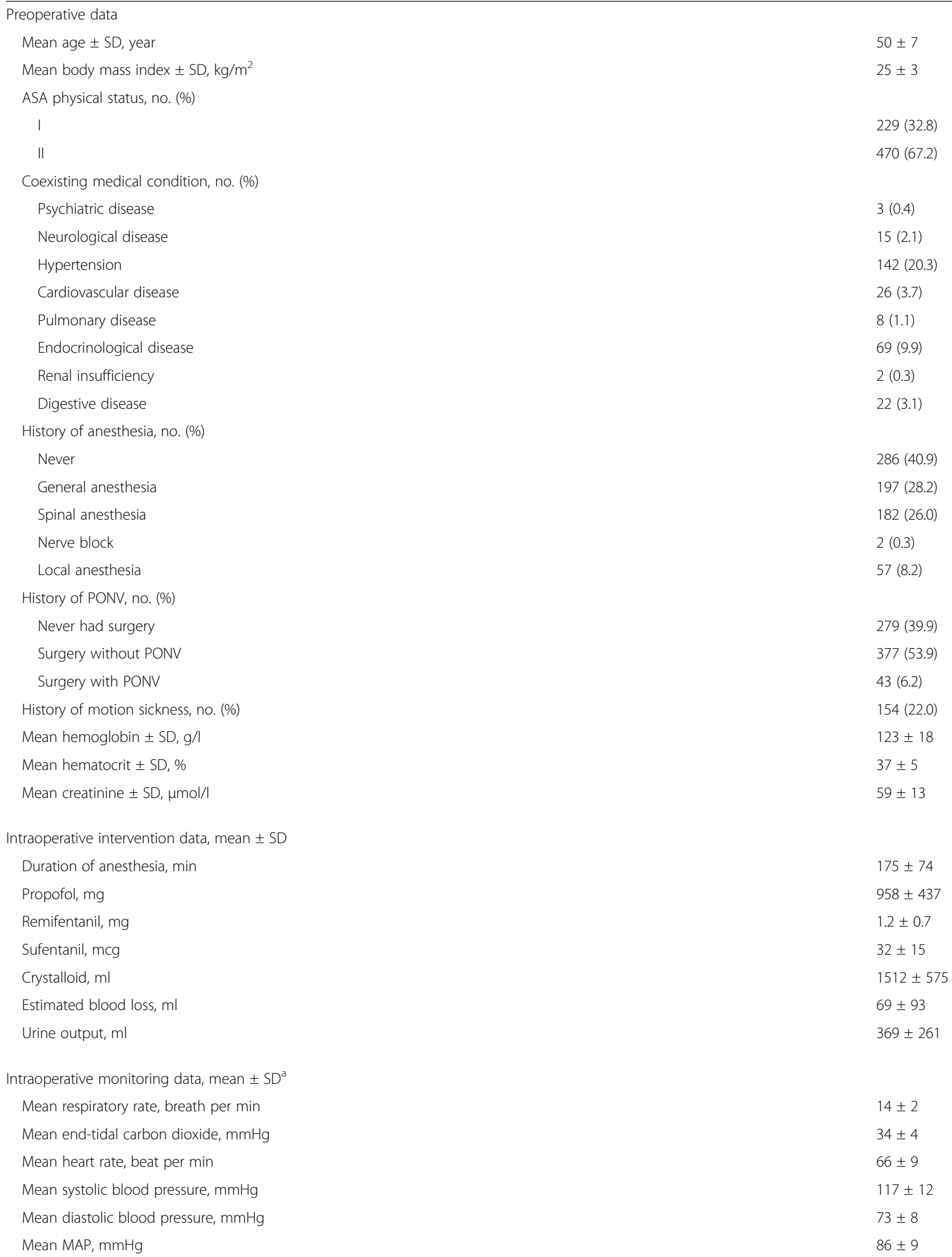


Table 2 Perioperative data $(n=699)$ (Continued)

\begin{tabular}{lc}
\hline Mean pulse oxygen saturation, \% & $100 \pm 1$ \\
Mean body temperature, ${ }^{\circ} \mathrm{C}$ & $36 \pm 1$ \\
Mean muscular tissue oxygen saturation, \% & $83 \pm 7$ \\
Postoperative QoR & $121 \pm 19$ \\
QoR-15 score, mean \pm SD & $122[109-135]$ \\
QoR-15 score, median [IQR] & 354 (50.6) \\
Number of patients with a QoR-15 $\geq 122$, no. (\%) &
\end{tabular}

$S D$ standard deviation, ASA American Society of Anesthesiologists, PONV postoperative nausea and vomiting, MAP mean arterial pressure, QoR quality of recovery, IQR interquartile range

${ }^{\mathrm{a}}$ For time-series data, we first removed those outliers defined as the data outside of the 0.5 th-99.5th percentile. The mean of all data within the 0.5 th-99.5th percentile was first derived for each patient. These means were then averaged to derive the mean for all patients

\section{Model discrimination}

Models' performance is presented in Table 3. When using the preoperative data only, all four models exhibited poor performance, with AUROCs ranging from 0.65 to 0.68 . The inclusion of the intraoperative intervention and/or monitoring data improved the performance of the deep leaning, logistic regression, and random forest models, but not the support vector machine model, which had an AUROC that remained in the range of 0.65-0.71.

In this study, performance was defined as indistinct if the AUROC's 95\% confidence interval (CI) overlaps. The deep learning model had indistinct performance when using the intraoperative intervention data only (AUROC, 0.79 ; $95 \% \mathrm{CI}, 0.75-0.82$ ), using the intraoperative monitoring data only (AUROC, 0.77; 95\% CI, 0.72-0.81), and using the preoperative, intraoperative intervention, and monitoring data combined (AUROC, 0.81; 95\% CI, $0.78-0.83)$.

The logistic regression model had indistinct performance when using the intraoperative intervention data only (AUROC, 0.78; 95\% CI, 0.74-0.82), using the intraoperative monitoring data only (AUROC, 0.72 ; 95\% CI, 0.68-0.77), and using the preoperative, intraoperative intervention, and monitoring data combined (AUROC, 0.77; 95\% CI, 0.70-0.85).

The random forest model had indistinct performance when using the intraoperative intervention data only (AUROC, 0.81; 95\% CI, 0.76-0.85) and using the preoperative, intraoperative intervention and monitoring data combined (AUROC, 0.82; 95\% CI, 0.78-0.87). In contrast, the performance was inferior when using the intraoperative monitoring data only (AUROC, 0.74; 95\% CI, 0.73-0.76).

\section{Model calibration}

The calibration plots and Brier scores are shown in Fig. 4. Compared to the logistic regression, support vector machine, and random forest models, the deep learning model exhibited better calibration when using the intraoperative monitoring data only (Brier score $=0.177$, Fig. 4a) and

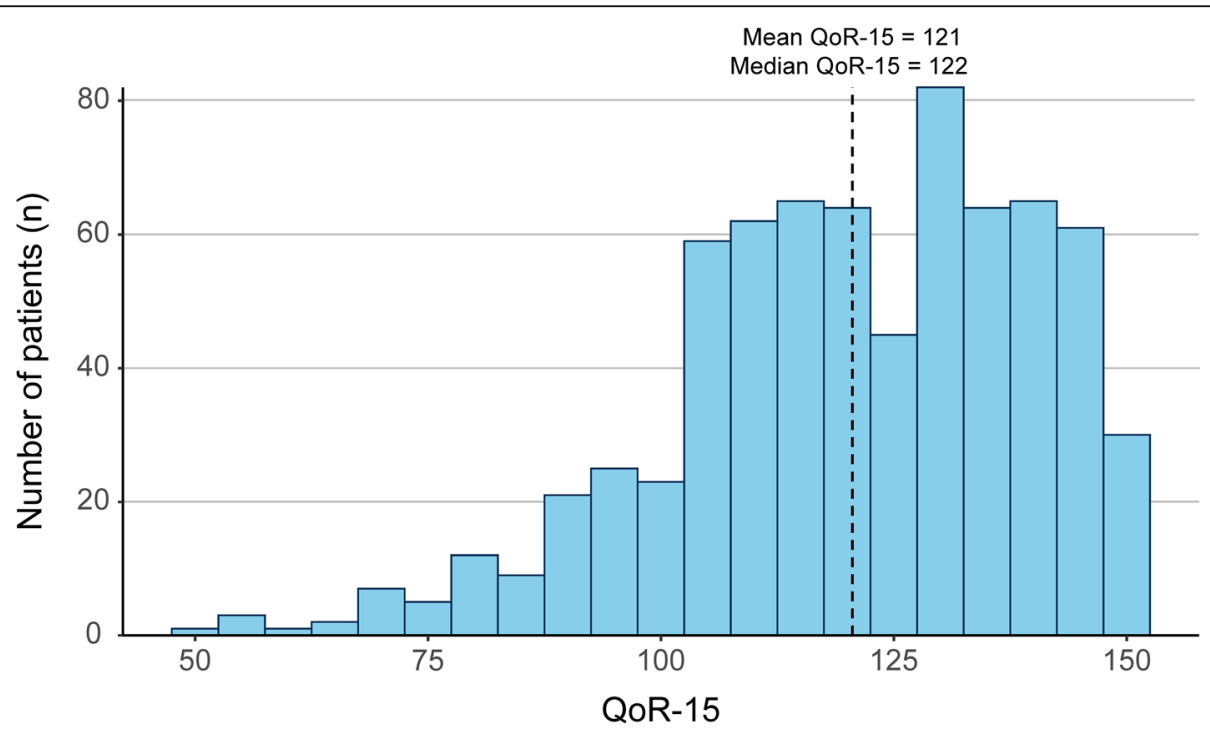

Fig. 3 QoR-15 distribution. QoR quality of recovery 
Table 3 Models' performance based on different datasets

\begin{tabular}{|c|c|c|c|c|c|}
\hline & Accuracy $^{a}$ & Sensitivity $^{a}$ & Specificity $^{a}$ & F1 score ${ }^{a}$ & AUROC \\
\hline \multicolumn{6}{|l|}{ Preoperative data } \\
\hline Deep learning & $0.61(0.57-0.65)$ & $0.61(0.54-0.68)$ & $0.61(0.50-0.71)$ & $0.60(0.56-0.64)$ & $0.65(0.62-0.67)$ \\
\hline Logistic regression & $0.63(0.59-0.66)$ & $0.62(0.59-0.65)$ & $0.63(0.56-0.71)$ & $0.62(0.60-0.65)$ & $0.68(0.66-0.70)$ \\
\hline Support vector machine & $0.61(0.56-0.66)$ & $0.51(0.40-0.62)$ & $0.70(0.59-0.81)$ & $0.56(0.49-0.63)$ & $0.65(0.60-0.70)$ \\
\hline Random forest & $0.62(0.60-0.65)$ & $0.59(0.49-0.70)$ & $0.66(0.59-0.72)$ & $0.60(0.55-0.66)$ & $0.68(0.65-0.70)$ \\
\hline \multicolumn{6}{|c|}{ Intraoperative intervention data } \\
\hline Deep learning & $0.74(0.70-0.79)$ & $0.73(0.66-0.80)$ & $0.74(0.61-0.87)$ & $0.74(0.71-0.77)$ & $0.79(0.75-0.82)$ \\
\hline Logistic regression & $0.76(0.71-0.81)$ & $0.77(0.73-0.80)$ & $0.76(0.64-0.88)$ & $0.76(0.73-0.79)$ & $0.78(0.74-0.82)$ \\
\hline Support vector machine & $0.59(0.54-0.64)$ & $0.50(0.41-0.59)$ & $0.67(0.55-0.80)$ & $0.54(0.48-0.60)$ & $0.65(0.61-0.68)$ \\
\hline Random forest & $0.73(0.67-0.79)$ & $0.75(0.71-0.78)$ & $0.72(0.58-0.86)$ & $0.74(0.69-0.78)$ & $0.81(0.76-0.85)$ \\
\hline \multicolumn{6}{|l|}{ Intraoperative monitoring data } \\
\hline Deep learning ${ }^{b}$ & $0.70(0.69-0.72)$ & $0.64(0.58-0.69)$ & $0.77(0.70-0.84)$ & $0.68(0.66-0.71)$ & $0.77(0.72-0.81)$ \\
\hline Logistic regression $^{c}$ & $0.69(0.63-0.75)$ & $0.68(0.64-0.72)$ & $0.69(0.56-0.83)$ & $0.68(0.64-0.73)$ & $0.72(0.68-0.77)$ \\
\hline Support vector machine ${ }^{c}$ & $0.62(0.58-0.66)$ & $0.61(0.56-0.66)$ & $0.63(0.52-0.75)$ & $0.62(0.59-0.64)$ & $0.68(0.65-0.71)$ \\
\hline Random forest ${ }^{c}$ & $0.61(0.57-0.65)$ & $0.83(0.72-0.94)$ & $0.40(0.21-0.58)$ & $0.68(0.66-0.69)$ & $0.74(0.73-0.76)$ \\
\hline \multicolumn{6}{|c|}{ Intraoperative monitoring data $+\mathrm{SmtO}_{2}$} \\
\hline Deep learning ${ }^{b}$ & $0.71(0.69-0.73)$ & $0.64(0.57-0.72)$ & $0.77(0.68-0.87)$ & $0.69(0.67-0.70)$ & $0.77(0.74-0.79)$ \\
\hline Logistic regression $^{c}$ & $0.69(0.63-0.75)$ & $0.68(0.64-0.72)$ & $0.69(0.54-0.85)$ & $0.69(0.65-0.72)$ & $0.73(0.68-0.78)$ \\
\hline Support vector machine ${ }^{c}$ & $0.67(0.64-0.70)$ & $0.63(0.57-0.69)$ & $0.70(0.61-0.79)$ & $0.65(0.63-0.68)$ & $0.71(0.67-0.76)$ \\
\hline Random forest ${ }^{c}$ & $0.65(0.60-0.70)$ & $0.87(0.79-0.95)$ & $0.44(0.27-0.61)$ & $0.71(0.70-0.73)$ & $0.78(0.73-0.82)$ \\
\hline \multicolumn{6}{|c|}{ Preoperative data + intraoperative monitoring data + intraoperative intervention data } \\
\hline Deep learning & $0.73(0.70-0.76)$ & $0.74(0.69-0.80)$ & $0.71(0.62-0.80)$ & $0.73(0.71-0.75)$ & $0.81(0.78-0.83)$ \\
\hline Logistic regression & $0.73(0.66-0.80)$ & $0.75(0.70-0.80)$ & $0.72(0.58-0.85)$ & $0.74(0.68-0.79)$ & $0.77(0.70-0.85)$ \\
\hline Support vector machine & $0.59(0.56-0.61)$ & $0.50(0.40-0.60)$ & $0.67(0.56-0.78)$ & $0.54(0.49-0.59)$ & $0.65(0.61-0.69)$ \\
\hline Random forest & $0.76(0.72-0.80)$ & $0.82(0.75-0.88)$ & $0.70(0.57-0.83)$ & $0.77(0.74-0.80)$ & $0.82(0.78-0.87)$ \\
\hline
\end{tabular}

Data are presented as mean $(95 \%$ confidence interval)

$A U R O C$ area under the receiver operating characteristic curve, $\mathrm{SmtO}_{2}$ muscular tissue oxygen saturation

${ }^{a}$ Calculated based on the decision threshold of 0.5

${ }^{\mathrm{b}}$ Based on time-series data

'Based on the maximum, minimum, mean, and standard deviation values of time-series data
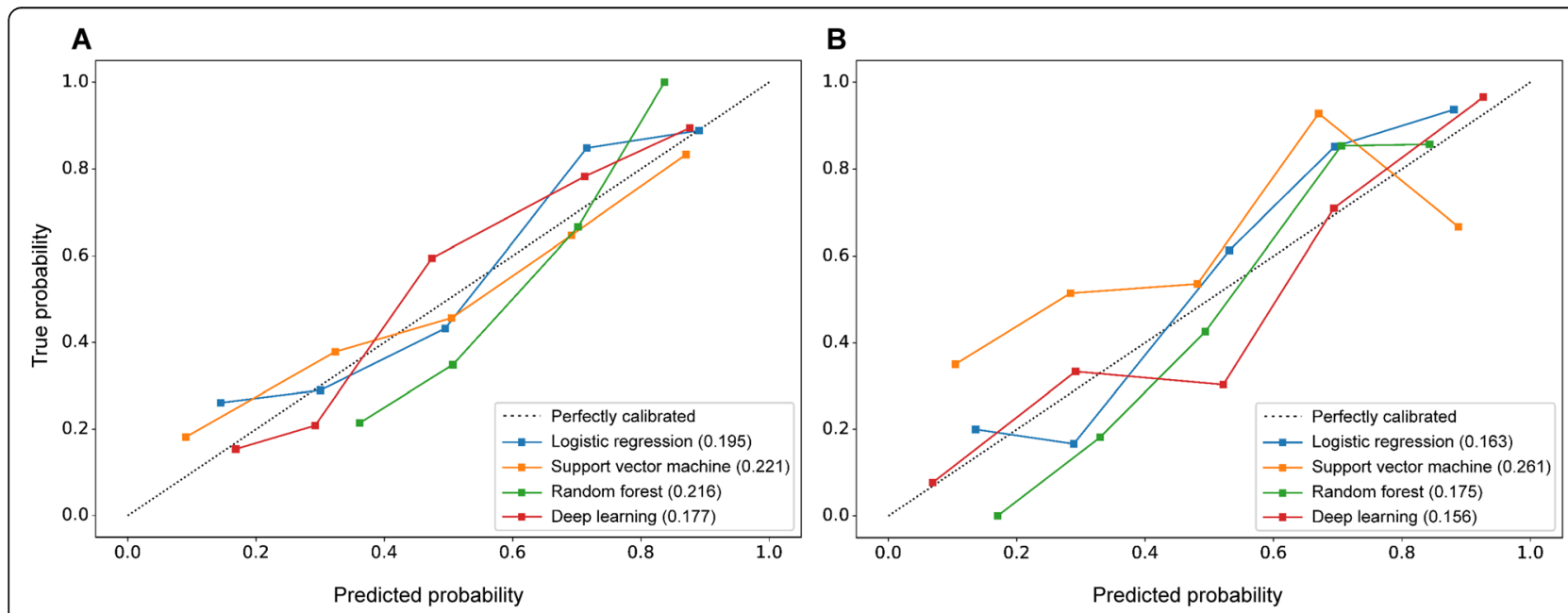

Fig. 4 Calibration plots and Brier scores of different models. Models based on intraoperative monitoring data (a) and based on preoperative data, intraoperative monitoring data, and intraoperative intervention data $(\mathbf{b})$ are presented 
when using the preoperative, intraoperative intervention, and monitoring data combined (Brier score $=0.156$, Fig. 4b).

\section{Feature importance}

SHAP values for the logistic regression and random forest models are presented in Fig. 5. We did not present SHAP values for the deep leaning and support vector machine models due to the unsuitability of using the SHAP method to explain the InceptionTime deep learning model and the poor performance of the support vector machine model in our study. Among features utilized in modeling, the dose of sufentanil administered during surgery appeared to have the most significant contribution to the prediction of the post-hysterectomy quality of recovery (Fig. 5b, d). A higher dose of sufentanil was associated with a higher likelihood of having an unsatisfactory quality of recovery (Fig. 5a, c).

\section{Class activation mapping}

Examples of class activation mapping are presented in eFigure 1 in Additional file 1. Overall, no specific parts of the temporal input appeared to have peculiar contributions.

\section{Discussion}

\section{Summary of findings}

We performed the first study investigating the prognostication of the quality of recovery using a deep learning model based on intraoperative time-series monitoring data in surgical patients. Our study has some unique findings. First, we found that the deep learning model based only on the intraoperative time-series monitoring data was able to predict the quality of recovery after laparoscopic hysterectomy. When using intraoperative monitoring data only, the performance of the deep learning model was better than the logistic regression and random forest models. This finding attests to the potential value of using the intraoperative time-series monitoring data for outcome prediction. Second, we found that inclusion of the intraoperative intervention and/or monitoring data significantly improved the performance of the deep learning, logistic regression, and random forest models compared to inclusion of the preoperative data only. This finding suggests that the performance of these models is input data-dependent. It also substantiates the close relationship between intraoperative management and postoperative outcomes. Third, we found that use of the preoperative, intraoperative intervention, and monitoring data combined did not significantly improve the models' performance compared to the use of intraoperative intervention data only or the use of the intraoperative monitoring data only. This finding suggests certain inherent associations among different datasets.

\section{Comparison with the current literature}

Machine learning recently began to make its footprint in the field of perioperative medicine (Hashimoto, Witkowski, Gao, Meireles, \& Rosman, 2020). Models based on deep learning algorithms have been developed for postoperative mortality prediction. Lee et al. developed a deep neural network model based on data from 59,985 patients to predict postoperative in-hospital mortality (Lee, Hofer, Gabel, Baldi, \& Cannesson, 2018). The AUROC of Lee et al.'s model was 0.88 (Lee et al., 2018). Fritz et al. developed a multipath convolutional neural network model based on data from 95,907 patients to predict 30-day postoperative mortality with an AUROC of 0.87 (Fritz et al., 2019).

Our study distinguished itself from these previous studies in the following aspects: (1) we targeted the quality of recovery in a relatively homogenous, young, and healthy female surgical patient population, while previous work targeted mortality in heterogeneous patient populations; (2) we investigated the models' performance based on different types of input data, while previous work did not; (3) our models were based on prospectively collected data, while previous work was based on retrospective data; (4) we used InceptionTime (Fawaz et al., 2019a, b), a state-of-the-art deep learning model, in our study, while previous work used different algorithms; and (5) we used high-frequency time-series monitoring data collected throughout the entire surgery, while Lee et al. did not use time-series data, and Fritz et al. used only time-series data collected over a random 60-min interval.

\section{Limitations}

Our study has limitations. First, our study was performed in relatively young and healthy female patients; therefore, caution is needed when generalizing our models to other patient populations (Moons et al., 2019). Second, the models' performances in our study were lower than that in Lee et al.'s study (Lee et al., 2018) and Fritz et al.'s study (Fritz et al., 2019); among the different potential causes for this inferiority, the most likely cause is the small sample size of our study. The model's accuracy becomes less when the sample size becomes smaller (Wisz et al., 2008). Third, it may seem arbitrary when we used a cutoff QoR-15 value of 122 to dichotomize the quality of recovery. However, this value was also adopted by a previous study (Kleif \& Gögenur, 2018) and is consistent with the mean and median QoR-15 scores of our patients (Li et al., 2020). Fourth, our models did not use some intraoperative data, e.g., the type and dose of vasoactive drugs. However, the vasoactive drugs may exert their characteristic footprints in time-series monitoring data. Thus, the use of timeseries monitoring data might have made up for the 


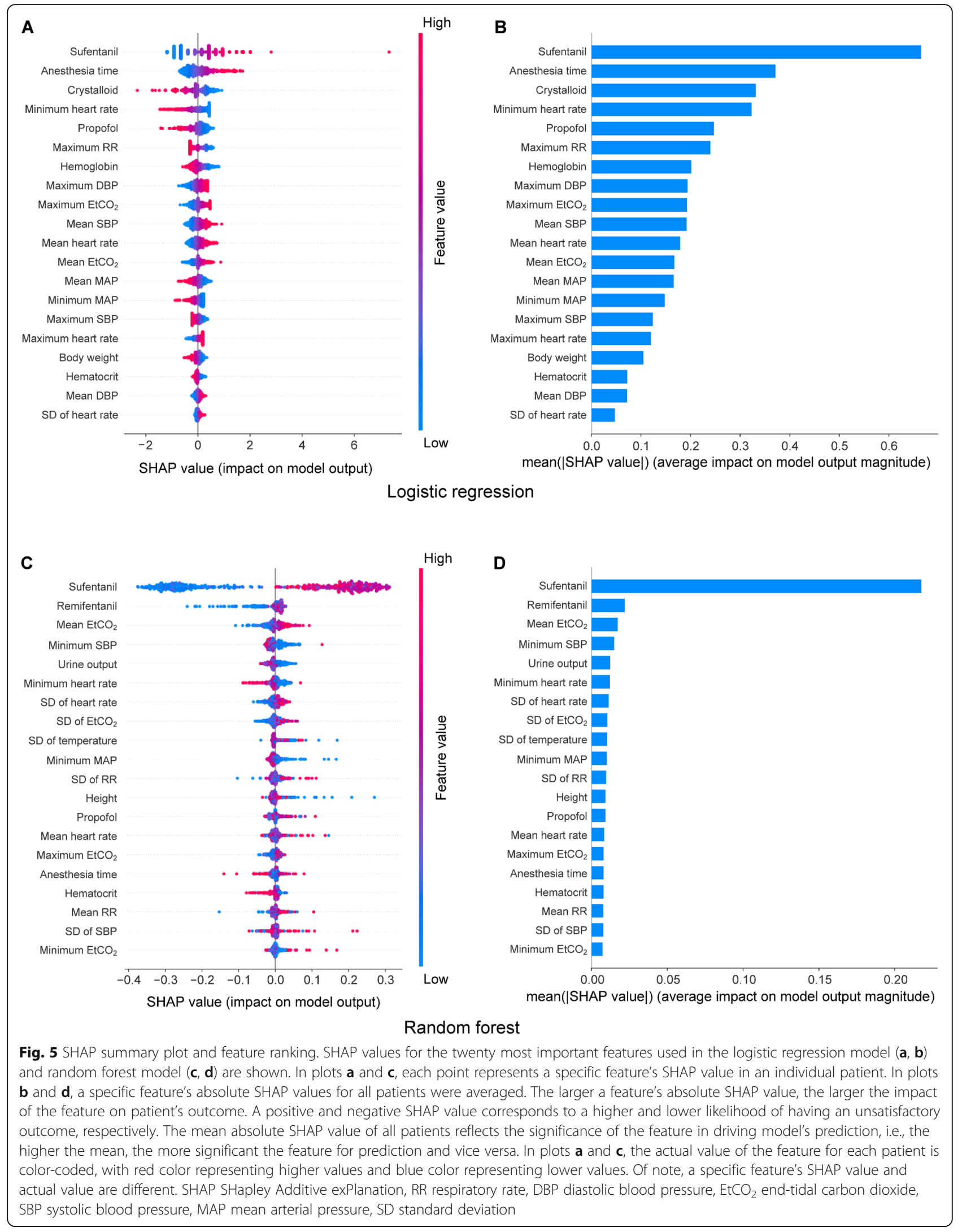


omission of certain intervention information in modeling. Fifth, we did not time stamp the intraoperative intervention data; instead, we used the total at the end of surgery in modeling. This approach might have ignored certain information that is valuable for modeling.

\section{Conclusions}

Deep learning based on the intraoperative time-series monitoring data can predict the quality of recovery after laparoscopic hysterectomy. The performance of the deep learning, logistic regression, and random forest models is input data-dependent. The inclusion of the intraoperative intervention and/or monitoring data significantly improved the models' performance compared to the inclusion of preoperative data only. These models may help clinicians identify at-risk patients, adjust perioperative care, and continuously improve the quality of clinical care. Our study should be regarded as a preliminary step towards accomplishing machine learning prediction based on intraoperative time-series monitoring data due to the various limitations discussed above. Moving forward, our models need to be validated using large-scale datasets with different patient populations.

\section{Supplementary Information}

The online version contains supplementary material available at https://doi. org/10.1186/s13741-021-00178-4.

Additional file 1: eTable 1. Performance of the deep learning model based on intraoperative monitoring data per different scaling methods. eTable 2. The maximum and minimum values used for continuous variable scaling. efigure 1. Class activation mapping in one patient. The degree of the contribution to prognostication is color coded, with red corresponding to a higher contribution and blue to a lower contribution. A. Deep learning model based on intraoperative monitoring data. B. Deep learning model based on preoperative data + intraoperative intervention data + intraoperative monitoring data

\section{Acknowledgements}

Support was provided by institutional and/or departmental sources

\section{Compliance with ethical standards}

Not applicable.

\section{Informed consent}

Not applicable.

\section{Authors' contributions}

Study conception/design: $X Z, K L$, and LM. Data analysis and model construction: $X Z$ and $K L$. Interpreting results: $X Z, K L$, and $L M$. Initial drafting of manuscript: $X Z$ and $L M$. Critical revision of manuscript: $X Z, K L, W W$, JX, and LM. Supervision: LM. The authors read and approved the final version of the manuscript.

\section{Funding}

No funding was received to conduct this study.

\section{Declarations}

Ethics approval and consent to participate

Not applicable.

\section{Consent for publication}

Not applicable.

\section{Competing interests}

Ke Liao and Wei Wang are Ricoh Software Research Center (Beijing) Co., Ltd. (Beijing, China) employees, but this work was performed independently from this position. The other authors declare no competing interests.

\section{Author details}

'Department of Anesthesiology, Yale University School of Medicine, 333 Cedar St, New Haven, CT 06520, USA. ${ }^{2}$ Department of Anesthesiology, The Second Xiangya Hospital, Central South University, Changsha, Hunan Province, China. ${ }^{3}$ Ricoh Software Research Center (Beijing) Co., Ltd., Beijing, China. ${ }^{4}$ School of Information Science, Japan Advanced Institute of Science and Technology, Nomi, Ishikawa, Japan.

Received: 16 November 2020 Accepted: 22 February 2021

Published online: 06 April 2021

\section{References}

Alba AC, Agoritsas T, Walsh M, Hanna S, lorio A, Devereaux P, et al. Discrimination and calibration of clinical prediction models: users' guides to the medical literature. JAMA. 2017:318(14):1377-84.

Bowyer A, Jakobsson J, Ljungqvist O, Royse C. A review of the scope and measurement of postoperative quality of recovery. Anaesthesia. 2014;69(11): 1266-78.

Coulter A, Locock L, Ziebland S, Calabrese J. Collecting data on patient experience is not enough: they must be used to improve care. BMJ. 2014; 348:g2225.

Fawaz HI, Forestier G, Weber J, Idoumghar L, Muller P-A. Deep learning for time series classification: a review. Data Mining Knowl Discov. 2019a;33(4):917-63.

Fawaz HI, Lucas B, Forestier G, Pelletier C, Schmidt DF, Weber J, et al. Inceptiontime: finding alexnet for time series classification. arXiv Preprint. 2019b;1909:04939.

Fritz BA, Cui Z, Zhang M, He Y, Chen Y, Kronzer A, et al. Deep-learning model for predicting 30-day postoperative mortality. Brit J Anaesthesia. 2019;123(5): 688-95.

Hashimoto DA, Witkowski E, Gao L, Meireles O, Rosman G. Artificial intelligence in anesthesiologycurrent techniques, clinical applications, and limitations. Anesthesiology. 2020;132(2):379-94.

Jammer I, Wickboldt N, Sander M, Smith A, Schultz MJ, Pelosi P, et al. Standards for definitions and use of outcome measures for clinical effectiveness research in perioperative medicine: European Perioperative Clinical Outcome (EPCO) definitionsA statement from the ESA-ESICM joint taskforce on perioperative outcome measures. Eur J Anaesthesiol. 2015;32(2):88-105.

Kleif J, Gögenur I. Severity classification of the quality of recovery-15 score-an observational study. J Surg Res. 2018;225:101-7.

Lee CK, Hofer I, Gabel E, Baldi P, Cannesson M. Development and validation of a deep neural network model for prediction of postoperative in-hospital mortality. Anesthesiology. 2018;129(4):649-62.

Li G, Tian D-D, Wang X, Feng X, Zhang W, Bao J, et al. Muscular tissue oxygen saturation and posthysterectomy nausea and vomiting the iMODIPONV randomized controlled trial. Anesthesiology. 2020;133(2):318-31.

Ljungqvist O, Scott M, Fearon KC. Enhanced recovery after surgery: a review. JAMA Surg. 2017;152(3):292-8. https://doi.org/10.1001/jamasurg.2016.4952.

Lundberg, S. M., \& Lee, S.-I. (2017). A unified approach to interpreting model predictions. Paper presented at the Advances in neural information processing systems.

Luo W, Phung D, Tran T, Gupta S, Rana S, Karmakar C, et al. Guidelines for developing and reporting machine learning predictive models in biomedical research: a multidisciplinary view. J Med Internet Res. 2016;18(12):e323. https://doi.org/10.2196/jmir.5870.

Mathis MR, Kheterpal S, Najarian K. Artificial intelligence for anesthesia: what the practicing clinician needs to knowmore than black magic for the art of the dark. Anesthesiology. 2018;129(4):619-22. 
Moons KG, Wolff RF, Riley RD, Whiting PF, Westwood M, Collins GS, et al. PROBAST: a tool to assess risk of bias and applicability of prediction model studies: explanation and elaboration. Ann Intern Med. 2019;170(1):W1-W33.

Myles PS, Bellomo R, Corcoran T, Forbes A, Peyton P, Story D, et al. Restrictive versus liberal fluid therapy for major abdominal surgery. New Engl I Med. 2018;378(24):2263-74.

Potdar K, Pardawala TS, Pai CD. A comparative study of categorical variable encoding techniques for neural network classifiers. Int J Comp Appl. 2017; 175(4):7-9.

Rufibach K. Use of Brier score to assess binary predictions. J Clin Epidemiol. 2010; 63(8):938-9.

Stark PA, Myles PS, Burke JA. Development and psychometric evaluation of a postoperative quality of recovery score the QoR-15. J Am Soc Anesthesiol. 2013;118(6):1332-40.

Wisz MS, Hijmans RJ, Li J, Peterson AT, Graham CH, Guisan A, NCEAS Predicting Species Distributions Working Group. Effects of sample size on the performance of species distribution models. Divers Distribut. 2008;14(5):76373.

Zhou, B., Khosla, A., Lapedriza, A., Oliva, A., \& Torralba, A. (2016). Learning deep features for discriminative localization. Paper presented at the Proceedings of the IEEE conference on computer vision and pattern recognition.

\section{Publisher's Note}

Springer Nature remains neutral with regard to jurisdictional claims in published maps and institutional affiliations.

Ready to submit your research? Choose BMC and benefit from:

- fast, convenient online submission

- thorough peer review by experienced researchers in your field

- rapid publication on acceptance

- support for research data, including large and complex data types

- gold Open Access which fosters wider collaboration and increased citations

- maximum visibility for your research: over $100 \mathrm{M}$ website views per year

At $\mathrm{BMC}$, research is always in progress.

Learn more biomedcentral.com/submissions 